\title{
Disease expression in juvenile polyposis syndrome: a retrospective survey on a cohort of 221 European patients and comparison with a literature-derived cohort of 473 SMAD4/BMPR1A pathogenic variant carriers
}

Robert Blatter, PhD ${ }^{1}$, Benjamin Tschupp, MMed ${ }^{1}$, Stefan Aretz, MD 2,3, Inge Bernstein, MD ${ }^{4}$, Chrystelle Colas, MD ${ }^{5,16}$, D. Gareth Evans, MD ${ }^{6}$, Maurizio Genuardi, MD ${ }^{7,8}$, Frederik J. Hes, MD, PhD 9 , Robert Hüneburg, $\mathrm{MD}^{3,10}$, Heikki Järvinen, $\mathrm{MD}^{11}$, Fiona Lalloo, MD ${ }^{6}$, Gabriela Moeslein, MD ${ }^{12}$, Laura Renkonen-Sinisalo, MD ${ }^{11}$, Nicoletta Resta, PhD ${ }^{13}$, Isabel Spier, MD ${ }^{2,3}$, Dora Varvara, MD ${ }^{13}$, Hans Vasen, MD ${ }^{14}$, Andrew R. Latchford, MD ${ }^{15}$ and Karl Heinimann, MD, PhD (i) ${ }^{1}$

Purpose: Juvenile polyposis syndrome (JPS) is a rare, autosomaldominantly inherited cancer predisposition caused in approximately $50 \%$ of cases by pathogenic germline variants in SMAD4 and $B M P R 1 A$. We aimed to gather detailed clinical and molecular genetic information on JPS disease expression to provide a basis for management guidelines and establish open access variant databases.

Methods: We performed a retrospective, questionnaire-based European multicenter survey on and established a cohort of SMAD4/ $B M P R 1 A$ pathogenic variant carriers from the medical literature.

Results: We analyzed questionnaire-based data on 221 JPS patients (126 kindreds) from ten European centers and retrieved literature-based information on 473 patients. Compared with BMPR1A carriers, SMAD4 carriers displayed anemia twice as often ( $58 \%$ vs. $26 \%$ ), and exclusively showed overlap symptoms with hemorrhagic telangiectasia (32\%) and an increased prevalence (39\% vs. 13\%) of gastric juvenile polyps. Cancer, reported in $15 \%$ of JPS patients (median age 41 years), mainly occurred in the colorectum (overall: $62 \%, S M A D 4: 58 \%, B M P R 1 A: 88 \%$ ) and the stomach (overall: $21 \%$; SMAD4: 27\%, BMPR1A: 0\%).

Conclusion: This comprehensive retrospective study on genotype-phenotype correlations in 694 JPS patients corroborates previous observations on JPS in general and SMAD4 carriers in particular, facilitates recommendations for clinical management, and provides the basis for open access variant SMAD4 and BMPR1A databases.

Genetics in Medicine (2020) 22:1524-1532; https://doi.org/10.1038/s41436020-0826-1

Keywords: polyposis; juvenile polyposis syndrome; colorectal cancer; genotype-phenotype correlation; hereditary hemorrhagic telangiectasia

\section{INTRODUCTION}

With an estimated incidence of 1:16,000 to 1:100,000, juvenile polyposis syndrome (JPS; OMIM 174900) represents a rare, autosomal-dominantly inherited cancer predisposition syndrome in which patients develop numerous juvenile type hamartomatous polyps (JPs) in the gastrointestinal (GI) tract and are at increased lifetime risk of $9-50 \%$ for GI cancers, mostly of the colon. ${ }^{1-3}$ The clinical diagnosis is established if an individual develops synchronously and/or metachronously five or more colorectal JPs, multiple JPs throughout the GI tract, or any number of JPs and a family history of juvenile polyposis. ${ }^{4}$ JPS is particularly prone to misdiagnosis (e.g., small juvenile polyps are often misdiagnosed as inflammatory polyps because they lack the diagnostic features of juvenile type polyps) and differentiation from PTEN hamartoma tumor syndrome (PHTS) can be challenging. Therefore,

\footnotetext{
${ }^{1}$ Institute for Medical Genetics and Pathology, University Hospital Basel, and Research Group Human Genomics, Department of Research, University of Basel, Basel, Switzerland; ${ }^{2}$ Institute of Human Genetics, University Hospital Bonn, Bonn, Germany; ${ }^{3}$ National Center for Hereditary Tumour Syndromes, University Hospital, Bonn, Germany; ${ }^{4}$ Department of Surgical Gastroenterology, Aalborg University Hospital, Aalborg, and Danish HNPCC Registry, Department of Surgical Gastroenterology, Hvidovre University Hospital, Hvidovre, Denmark; ${ }^{5}$ Department of Oncogenetics and Angiogenetics, Pitie-Salpetriere Hospital, Sorbonne Université, Paris, France; ${ }^{6}$ Department of Genomic Medicine, Manchester Universities NHS Foundation Trust and Division of Evolution and Genomic Sciences, University of Manchester, Manchester, United Kingdom; ${ }^{7}$ Fondazione Policlinico Universitario A. Gemelli IRCCS, UOC Genetica Medica, Rome, Italy; ${ }^{8}$ Università Cattolica del Sacro Cuore, Istituto di Medicina Genomica, Rome, Italy; ${ }^{9}$ Department of Clinical Genetics, Leiden University Medical Center, Leiden, The Netherlands; ${ }^{10}$ Department of Internal Medicine I, University Hospital Bonn, Bonn, Germany; ${ }^{11}$ Department of Surgery, Helsinki University Central Hospital, Helsinki University, Helsinki, Finland; ${ }^{12}$ Center for Hereditary Tumors, HELIOS Klinikum Wuppertal, University Witten-Herdecke, Wuppertal, Germany; ${ }^{13}$ Division of Medical Genetics, Department of Biomedical Sciences and Human Oncology (DIMO), University of Bari “Aldo Moro”, Bari, Italy; ${ }^{14}$ Department of Gastroenterology and Hepatology, Leiden University Medical Centre, Leiden, The Netherlands; ${ }^{15}$ Polyposis Registry, St. Mark's Hospital, Harrow, United Kingdom; ${ }^{16}$ Present address: Department of Genetics, Institut Curie, Université de Recherche Paris Sciences et Lettres, Paris, France. Correspondence: Andrew R. Latchford (andrew.latchford@nhs.net) or Karl Heinimann (karl.heinimann@usb.ch)

These authors contributed equally: Robert Blatter, Benjamin Tschupp.
}

Submitted 27 September 2019; revised 27 April 2020; accepted: 27 April 2020

Published online: 13 May 2020 
multigene panel diagnostics in patients with clinical suspicion of PHTS should also include SMAD4 and BMPR1A.

In $45-55 \%$ of all JPS patients the clinical diagnosis can be confirmed by identification of a germline pathogenic variant $(\mathrm{PV})$ in either the SMAD4 (Locus Reference Genomic [LRG] ID LRG_318t1) or the BMPR1A (LRG ID LRG_298t1) gene, with the majority consisting of missense and nonsense variants, small insertions/deletions, and large genomic deletions. $^{5-7}$ Both proteins belong to the transforming growth factor- $\beta$ (TGF- $\beta$ )/bone morphogenetic protein (BMP) superfamily of molecules with SMAD4 serving as central mediator of TGF- $\beta$ and BMP signaling. ${ }^{8}$ While SMAD4 loss-of-function alterations have been associated with tumorigenesis as well as with a juvenile polyposis-hereditary hemorrhagic telangiectasia overlap syndrome (JPS-HHT; OMIM $175050^{9}$ ), SMAD4 de novo gain-of-function variants result in autosomaldominant Myhre syndrome, a connective tissue disorder with multisystem involvement and intellectual disability (OMIM $\left.139210^{10}\right)$. Moreover, microdeletions encompassing both the $B M P R 1 A$ and the PTEN gene loci on chromosome 10q22-q23 have been described in patients presenting with early-onset JPS and features of PTEN hamartoma tumor syndrome, the severity depending on the extent of the deletion. ${ }^{11}$ The pathogenetic events behind SMAD4- and BMPR1A-associated juvenile polyposis are still poorly understood; only scarce data are available on the putative roles of these genes as tumor suppressors and the nature of the respective somatic alterations (2nd hit) involved in polyp formation. ${ }^{12-15}$

Despite being known for more than 50 years ${ }^{16}$ clinical guidelines for JPS, due to its rarity, still essentially depend on clinical and genetic information gathered from single series/ center reports, mostly focused on SMAD4 PV carriers, and expert opinion. ${ }^{17}$ Additionally, there exists no comprehensive, open access database on genetic variants in SMAD4 or BMPR1A. The aims of this retrospective study were to describe the clinical and molecular genetic features from a European, multicenter survey on JPS patients, compare them with literature-derived data on SMAD4/BMPR1A PV carriers, and provide recommendations for management on the basis of both new and previously published data. In addition, the data establish the basis for SMAD4 and BMPR1A Leiden Open Variant Databases (LOVD).

\section{MATERIALS AND METHODS \\ European collaborative study (ECS) group Enrollment and data collection}

Ten medical centers from eight European countries participated in this retrospective, European collaborative study (ECS) on JPS and contributed patient data in an anonymized fashion based on a questionnaire that specifically asked for clinical, familial, and genetic details (Supplementary Table 1 and Supplementary Fig. A). The study was conducted with the approval of the Ethikkommission beider Basel ("Basler Studie ueber familiaere Tumorkrankheiten," number 258/05) and all collaborating centers had obtained local approval for the study according to national guidelines, adhering to the principles set out in the Declaration of Helsinki. Informed consent was obtained from all patients. Only patients who fulfilled the clinical criteria for JPS and in whom a disease causing, i.e., likely pathogenic (class 4) or pathogenic (class 5) SMAD4 or BMPR1A germline variant could be identified were submitted. Thirty JPS patients had to be excluded from the study because of incomplete genetic and/or clinical data. The final ECS group comprised 221 patients from 126 kindreds (1 to 9 patients per kindred).

\section{Literature-based SMAD4/BMPR1A PV carrier (LBSB) group Search and selection criteria}

To retrieve all published SMAD4/BMPR1A carriers of PVs reported in the medical literature, a comprehensive search for entries between 1 January 1998 and 31 December 2018 was performed using six databases: CINAHL, EMBASE, MEDLINE/PubMed, PsycINFO, Psyndex, and Web of Science. Each database was searched individually for genotype and phenotype characteristics using both free text searches as well as entry term searches (Supplementary Fig. B). Following the selection process depicted in Supplementary Fig. C and exclusion of (1) patients with promoter or unspecified SMAD4/BMPR1A variants, (2) patients with SMAD4associated Myhre syndrome $(n=8)$, and (3) ECS group patients having already been published $(n=39), 628$ SMAD4/ $B M P R 1 A$ variant carriers could be retrieved. Applying variant classification criteria based on the American College of Medical Genetics and Genomics (ACMG) standards and guidelines $^{18}$ a total of 473 patients (249 SMAD4, 224 $B M P R 1 A$ ) carrying pathogenic (i.e., class 4 and 5) gene variants were included, and phenotype data, as for the ECS group, were gathered, where available, from the pertinent publications for comparison.

\section{Categorization of polyp numbers reported}

Information on polyp numbers was summarized in three categories: "few," "multiple," and "massive." "Few" encompasses descriptions such as "few," "some," or 1-4 polyps; "multiple" such as "multiple," "numerous," "several," "many," or 5-99 polyps; and "massive" containing "massive," "diffuse," "carpeted," "lots," or $\geq 100$ polyps.

\section{Statistical methods}

Statistical comparison of patients' features, encompassing phenotypic characteristics and molecular status, was done using Chi-square and Fisher's exact tests for categorical variables (e.g., gender, presence of symptoms), or Student's $t$ test for continuous variables (e.g., age at diagnosis), with all of the probabilities reported as two-tailed $P$ values, considering $p<0.05$ to be statistically significant. All calculations were done either in Microsoft Excel 2016 or Stat View v.4.5 (Abacus Concepts).

\section{RESULTS}

The 221 ECS patients (sex ratio: m:f=105:116) from 126 kindreds consisted of 127 patients with a germline PV in the 
SMAD4 gene (57.5\%; 71 kindreds; 56 nonindex) and 94 patients with a PV in the BMPR1A gene (42.5\%; 55 kindreds; 39 nonindex). Having excluded previously published ECS patients, the LBSB group consisted of 473 patients (224 $B M P R 1 A ; 249$ SMAD4) with individual phenotypic information ascertained from 232 (49.1\%) PV carriers (sex ratio [ $n=$ 160]: $m: f=80: 80)$.

\section{Age at diagnosis}

The median age at JPS diagnosis of all ECS patients was 25.0 years (interquartile range [IQR] 28.0, range 0.2-86 years) with a similar age at diagnosis for SMAD4 (28.0 years) and BMPR1A (24.5 years) PV carriers (Supplementary Table 2). A significant difference in age at diagnosis was seen between index patients (19.5 years; IQR 23.5, range 0.6-86 years) compared with other family members, referred to as nonindex patients (32.0 years; IQR 30.5, range $0.2-76$ years; $p=0.0017)$.

While median age at JPS diagnosis was similar for LBSB patients in general (23.0 years, IQR $27.0 ; n=104)$ and for SMAD4 carriers (26.0 years, IQR $26.5 ; n=71$ ), carriers of large cytogenetic alterations, i.e., microdeletions at $10 \mathrm{q} 23$ encompassing the BMPR1A and PTEN gene loci, displayed a significantly younger age ( 1.5 years, IQR $2.6 ; n=8)$ compared with carriers of other BMPR1A pathogenic variants (23.0 years, IQR 26.0; $n=26 ; p<0.001)$. This was also observed in the five ECS index patients with 10q23 microdeletions whose median age at diagnosis was 6.0 years (IQR 5.0) compared with carriers of other types of BMPR1A variants (21.0 years; IQR $19.0 ; n=48 ; p=0.009$ ).

\section{Symptoms at presentation}

The most common symptoms reported in ECS index patients were rectal bleeding (43\%) and anemia (47\%). Whereas the former was nearly equally frequent in SMAD4 (41\%) and BMPR1A (46\%) PV carriers, anemia was reported twice as often in SMAD4 carriers (58\% vs. $26 \%, p=0.0003$; Table 1), who also exhibited symptoms of hereditary hemorrhagic telangiectasia in $32 \%$ (13/41). In ECS nonindex patients, these symptoms were significantly less common in SMAD4 (14\%; $11 \% ; p=0.006)$ and absent in BMPR1A PV carriers $(0 \%$ for both; $p<0.0001)$. Symptoms associated with JPS-HHT, such as epistaxis, telangiectasia, or other types of arteriovenous malformations, were exclusively reported in SMAD4 PV carriers $(24 \%, 21 \%$, and $14 \%$ of index patients, respectively) but not in BMPR1A PV carriers (0\%). A similar pattern was observed in LBSB patients (SMAD4 [ $n=55]: 16.1 \%, 10.8 \%$, and $12.6 \%$; BMPR1A: 0\%).

Congenital heart defects (CHD), mainly ventricular and atrial septal defects, were reported in 3 (4.2\%) SMAD4 and 5 (9.1\%) BMPR1A index patients $(p=0.29$; Table 1). Overall, $\mathrm{CHD}$ was more frequently stated in $B M P R 1 A$ compared with

Table 1 Symptoms at presentation.

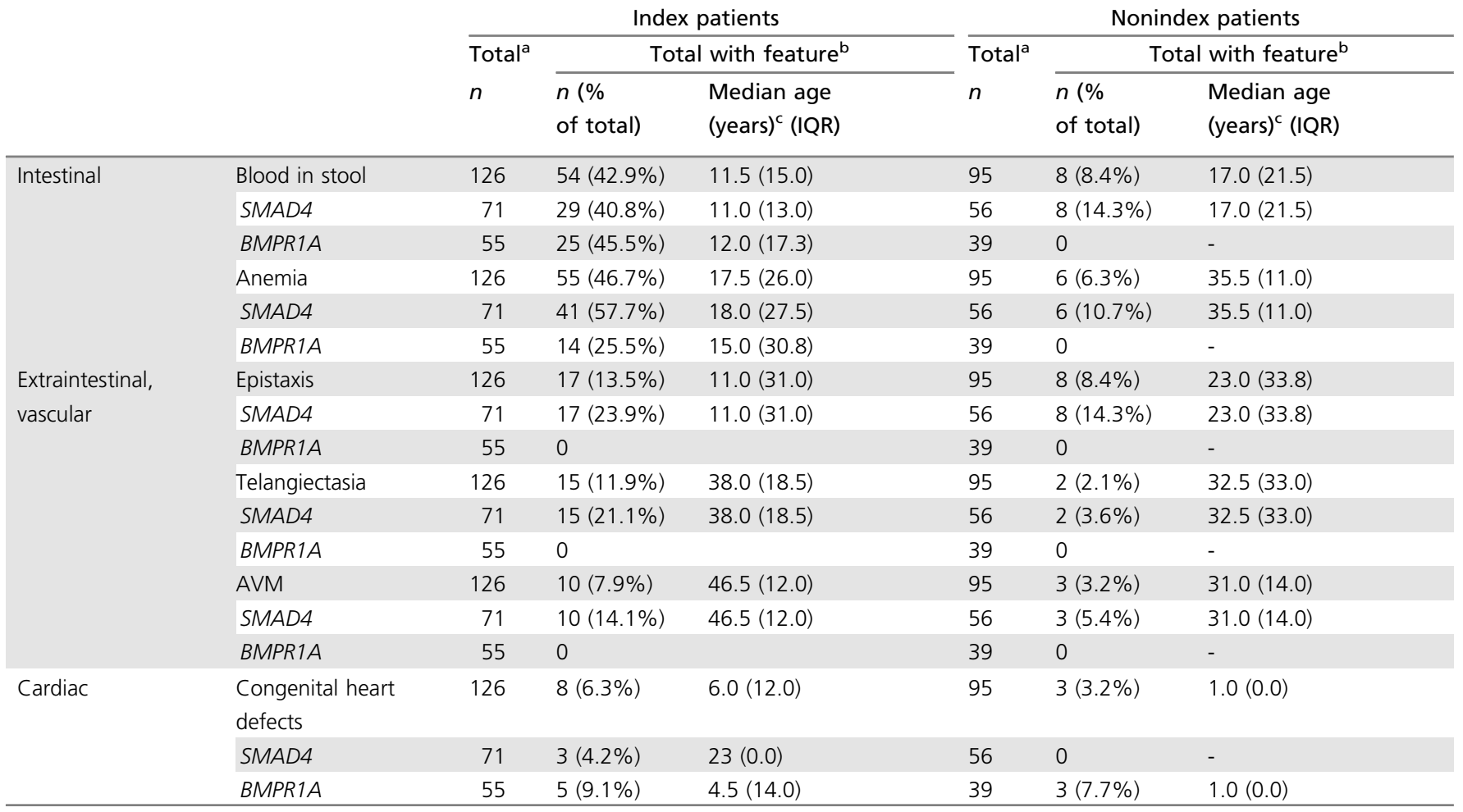

$A V M$ arteriovenous malformation, $I Q R$ interquartile range.

${ }^{\text {a }}$ Total number of patients.

${ }^{b}$ Total number of index or nonindex patients who presented with the respective symptom.

cDue to missing age at diagnosis the calculation of the median age is often based on fewer than the total number of patients with the respective feature. 
Table 2 Polyp occurrence in the Gl tract.

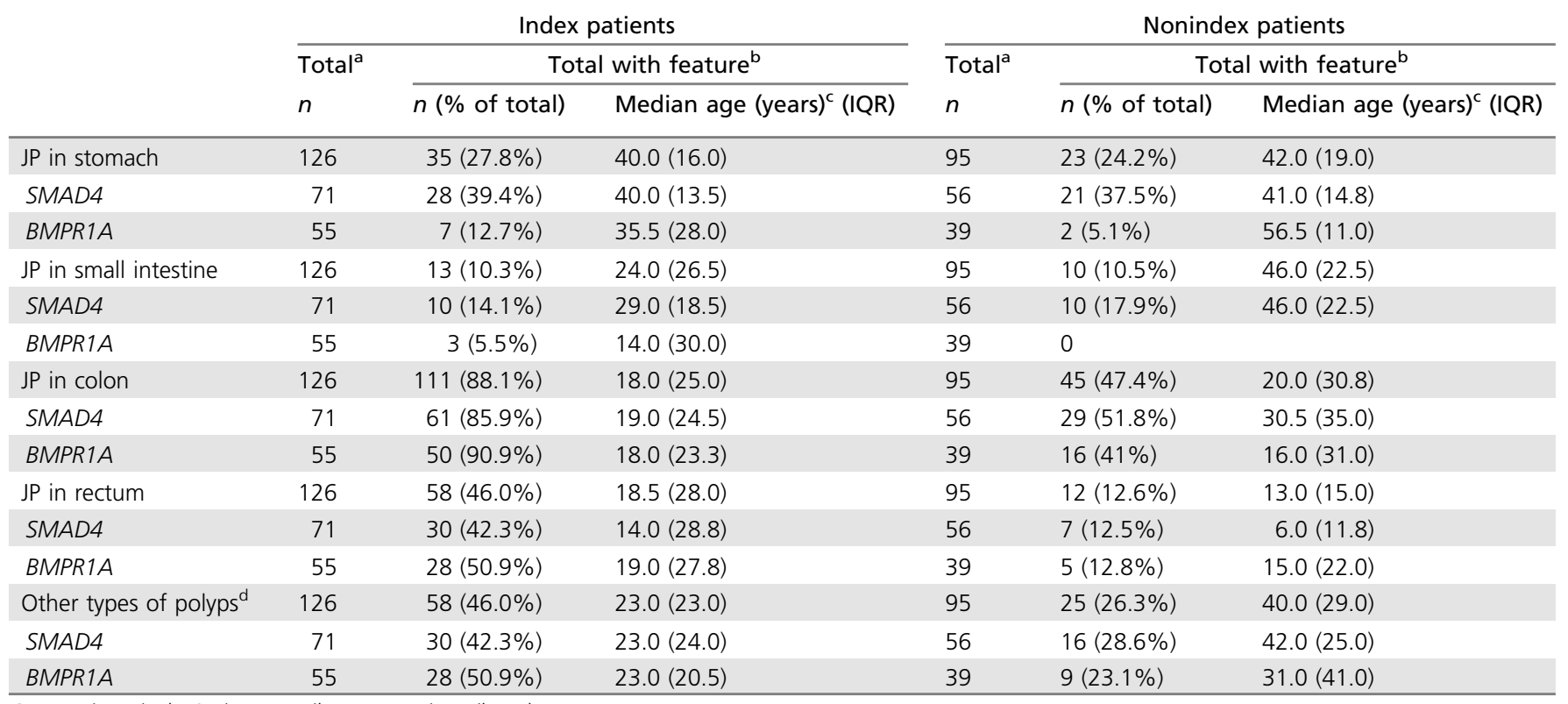

Gl gastrointestinal, IQR interquartile range, JP juvenile polyp.

aTotal number of patients.

${ }^{b}$ Total number of index or nonindex patients who presented with the respective symptom.

${ }^{c}$ Due to missing age at diagnosis the calculation of the median age is often based on fewer than the total number of patients with the respective feature.

${ }^{\mathrm{d}}$ Adenomas, serrated and other polyps.

SMAD4 PV carriers (8.5\% [8/94] vs. $2.4 \%[3 / 127] ; p=0.06)$, which was also the case in the LBSB group $(4.0 \%[9 / 224]$ vs. $0.4 \%$ [1/249]; $p=0.008)$. The small numbers precluded any meaningful statistical/genotype-phenotype analysis; we did not, however, observe an overrepresentation of a specific type of PV or protein domain affected. Aortic aneurysms were exclusively reported in SMAD4 PV carriers (ECS group: 2 [1.6\%], LBSB group: 1 [0.4\%]).

Few ECS and LBSB patients displayed other symptoms such as macrocephaly ( $n=11$, with 9 of them carrying a $10 \mathrm{q} 32$ microdeletion encompassing the BMPR1A and PTEN gene loci), failure to thrive $(n=9)$, and protein-losing enteropathy (5). Except for a LBSB group patient with a 10q32 microdeletion, no further cases of cleft palate or polydactyly were reported.

\section{Polyp occurrence in the GI tract}

Juvenile polyps in the colorectum

A high prevalence of juvenile polyps (JPs) in the colon was reported in ECS index patients (BMPR1A: 91\% and SMAD4: $86 \%)$, essentially mirrored by the LBSB group (96\% [42/44] and $93 \%$ [69/74]), whereas this was significantly less frequently the case for ECS nonindex patients (41\% and 52\%, respectively; $p<0.0001$; Table 2). JPs were more frequently reported in the proximal than the distal colon, both in index $(40 / 70 ; 57.1 \%)$ and clinically affected nonindex $(13 / 19 ; 68.4 \%)$ patients (Table 3). The load of colonic JPs was similar in both groups, ECS and LBSB, as well as in the gene-specific subgroups, with most index patients presenting with multiple (5 to 99) JPs (Table 3). In the ECS group, median age at diagnosis was similar for index and nonindex patients $(18.0$ years and 20.0 years). Interestingly, BMPR1A index and nonindex patients displayed a similar median age at diagnosis, compared with SMAD4 carriers where nonindex patients were diagnosed about 11.5 years later. Unfortunately, for most LBSB patients specific information regarding age at diagnosis and polyp occurrence could not be retrieved from the respective publications, which precluded meaningful calculations in the corresponding LBSB subgroups.

Compared with those in the colon, rectal JPs in the ECS group overall were significantly less frequently reported (70.6\% $[n=156]$ vs. $31.7 \%[n=70] ; p<0.01)$ and appeared to be somewhat more prevalent in BMPR1A index patients (Table 2). In nonindex patients median age at diagnosis of rectal JP was lower (13.0 years) compared with colonic JP (20.0 years, $p=0.15$; Table 4 ). Rectal polyp load was similar among BMPR1A and SMAD4 PV carriers, with the majority of patients presenting with few $(<5)$ to multiple (5 to 100) JPs (Table 3).

\section{Juvenile polyps in the stomach and in the small bowel}

As specific information regarding the presence of JP in the upper GI tract is largely missing in the LBSB patients, we only report the findings in the ECS patients. A clear genotype-phenotype correlation was observed for gastric juvenile polyposis, which was significantly more frequent in SMAD4 than BMPR1A PV carriers, both in index and nonindex patients (SMAD4 $39 \%$ and $38 \%$ vs. BMPR1A $13 \%$ and $5 \% ; p=0.001$ and 0.0002 , respectively; Table 2). JP formation in the stomach was also more severe in SMAD4 
Table 3 Number of juvenile polyps according to GI tract site.

\begin{tabular}{|c|c|c|c|c|c|c|}
\hline & \multicolumn{3}{|c|}{ Index patients } & \multicolumn{3}{|c|}{ Nonindex patients } \\
\hline & Total & SMAD4 & BMPR1A & Total & SMAD4 & $B M P R 1 A$ \\
\hline Stomach & 31 & 24 & 7 & 17 & 15 & 2 \\
\hline Multiple & 18 & $17(71 \%)$ & $1(14 \%)$ & 6 & $6(40 \%)$ & 0 \\
\hline Massive & 4 & $4(17 \%)$ & 0 & 4 & $4(27 \%)$ & 0 \\
\hline Small intestine & 10 & 7 & 3 & 9 & 9 & 0 \\
\hline Multiple & 4 & $2(29 \%)$ & $2(67 \%)$ & 4 & $4(44 \%)$ & $0(\%)$ \\
\hline Massive & 0 & 0 & 0 & 0 & 0 & 0 \\
\hline Colon & 92 & 49 & 43 & 37 & 23 & 14 \\
\hline Few & 11 & $7(14 \%)$ & $4(9 \%)$ & 9 & $5(22 \%)$ & $4(29 \%)$ \\
\hline Multiple & 69 & $33(68 \%)$ & $36(84 \%)$ & 27 & $17(74 \%)$ & $10(71 \%)$ \\
\hline Massive & 1 & $1(4 \%)$ & 0 & 0 & 0 & 0 \\
\hline Distal colon & 30 & 20 & 10 & 6 & 3 & 3 \\
\hline Few & 14 & $9(45 \%)$ & $5(45 \%)$ & 2 & 0 & $2(67 \%)$ \\
\hline Multiple & 15 & $10(50 \%)$ & $5(55 \%)$ & 4 & $3(100 \%)$ & $1(33 \%)$ \\
\hline Massive & 1 & $1(5 \%)$ & 0 & 0 & 0 & 0 \\
\hline Rectum & 38 & 20 & 18 & 8 & 3 & 5 \\
\hline Few & 16 & $11(50 \%)$ & $5(28 \%)$ & 6 & $3(100 \%)$ & $3(60 \%)$ \\
\hline Multiple & 22 & $9(50 \%)$ & $13(72 \%)$ & 2 & 0 & $2(40 \%)$ \\
\hline Massive & 0 & 0 & 0 & 0 & 0 & 0 \\
\hline Other polyps & 48 & 25 & 23 & 16 & 12 & 4 \\
\hline
\end{tabular}

G/ gastrointestinal.

pathogenic variant carriers since most $B M P R 1 A$ PV carriers $(86 \%)$ presented with only few $(<5)$ polyps, whereas most SMAD4 PV carriers $(71 \%)$ presented with multiple JPs or even a massive $(\geq 100)$ number of JPs $(17 \% ; p=0.0001$; Table 3). Combining the data from index and nonindex patients, who displayed similar JP loads, the difference remained statistically significant $(p=0.0002)$. JP formation in the small intestine was also more often reported in SMAD4 (15.7\%) than BMPR1A PV carriers (3.2\%; Table 2).

\section{Age at diagnosis of JP in the GI tract}

The median age at JP diagnosis in the different sections of the GI tract did not significantly differ between ECS-SMAD4 and ECS$B M P R 1 A$ PV carriers. A significant difference was only observed for index patients with stomach polyps where the reported median age at diagnosis was roughly 20 years later (40.0 years) compared with colonic (18.0 years; $p<0.0001$; Table 2 ).

\section{Other polyps}

In $37.6 \%$ of ECS and $82.2 \%$ of LBSB patients other types of polyps were reported, mostly adenomas and hyperplastic polyps, with similar frequencies in SMAD4 and BMPR1A PV carriers (ECS: $36.2 \%$ and 39.4\%; LBSB: $72.7 \%$ and $96.6 \%$, respectively; Table 2).

\section{Cancer occurrence}

Cancer was reported in 15.4\% (34/221) of ECS patients (index patients: 15.1\% [19/126]; nonindex patients: $15.8 \%$ [15/95], and 11.0\% [52/473] of LBSB patients; Table 4). In most ECS patients $(78.1 \%, 25 / 32)$ the cancer diagnosis was either accompanied or preceded by the diagnosis of colonic JPs. A notable exception was found in three ECS-SMAD4 pathogenic variant carriers, two index and one nonindex, who presented with cancer and JPs in the stomach/small intestine, but none in the colorectum; instead of JPs, two of them were reported to have multiple colorectal adenomas or hyperplastic polyps. The overall median age at cancer diagnosis was 41 years, which is 23 years later compared with the diagnosis of colon JPs $(18.5$ years, $p<0.0001)$ and similar to that of gastric JP (40 years). Overall, cancer was significantly more frequently observed in ECS-SMAD4 $(20.5 \%, n=26)$ compared with ECS-BMPR1A PV carriers 
Table 4 Cancer occurrence in JPS patients.

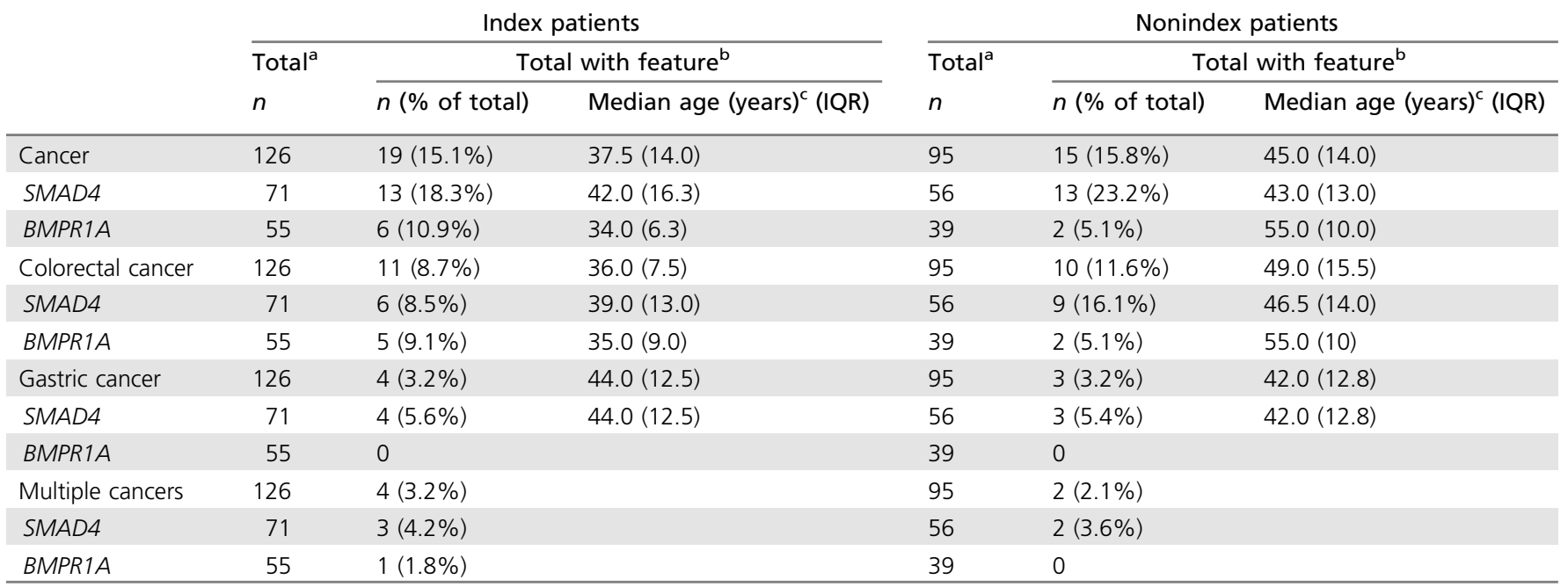

IQR interquartile range, JPS juvenile polyposis syndrome.

${ }^{a}$ Total number of patients.

${ }^{b}$ Total number of index or nonindex patients who presented with the respective symptom.

'Due to missing age at diagnosis the calculation of the median age is often based on fewer than the total number of patients with the respective feature.

$(8.5 \% ; n=8 ; p=0.015)$, which could be attributed to the difference in cancer occurrence among nonindex patients ( $23 \%$ vs. $5 \%$, respectively; $p=0.022$ ).

Colorectal cancer $(n=21$; median age at diagnosis: 38 years; range 19-67 years) was reported in $11.8 \%(15 / 127)$ of ECS-SMAD4 and 7.4\% (7/94) of ECS-BMPR1A carriers (Table 4). It accounted for $61.8 \%$ (21/34) of all cancers reported, representing $57.7 \%(15 / 26)$ of all SMAD4 and $87.5 \%$ $(7 / 8)$ of all BMPR1A cancers. Eighty percent (12/15) of $S M A D 4$ variants were nonsense/frameshift type compared with $28.6 \%(2 / 7)$ in BMPR1A carriers $(p=0.052)$. Gastric cancer ( $n=7$; all SMAD4; median age at diagnosis: 44 years; range $38-55$ years) was reported in SMAD4 carriers only (5.5\%; 7/127) and accounted for 20.6\% (7/34) of all cancers reported and for $26.9 \%(7 / 26)$ of all SMAD4 tumors. Similar observations were made in the LBSB group where colorectal and gastric cancer accounted for 63\% $(n=12)$ and $15.8 \%$ $(n=3$; all SMAD4) of the cancer burden. Few other cancers reported in the ECS group were located in the small intestine $(n=1)$, thyroid $(n=2)$, lung $(n=1)$, and cancer of unknown origin $(n=1)$.

Six (2.7\%) ECS patients were reported to have developed metachronous cancers (Table 4). In three of them both cancers had occurred in the GI tract (three colorectal, three gastric, and one cancer of the small intestine), whereas three, each with colorectal cancer, had additionally developed lung, pancreatic, and thyroid cancer, respectively.

\section{Pathogenic variant type frequencies}

Overall, frameshift, nonsense, and missense variants accounted for the majority of pathogenic SMAD4 (72.9\%) and BMPR1A (61.8\%) alterations in the ECS as well as the LBSB group (SMAD4: 79.9\%; BMPR1A: 70.8\%; Supplementary Table 3). Only large genomic, i.e., single or multiexon deletions in SMAD4 were significantly overrepresented in the ECS compared with the LBSB group (21.4\% vs. $6.8 \%$; $p=$ $0.003)$. De novo variant occurrence was suspected in 10 (7.9\%; 8 SMAD4, 2 BMPR1A) of 126 ECS index patients as well as in 26 (5.5\%; 11 SMAD4, 15 BMPR1A) of 473 LBSB pathogenic variant carriers.

As detailed above, statistically significant genotype-phenotype correlations were identified with regard to younger age at diagnosis in carriers of 10q23 microdeletions encompassing the $B M P R 1 A$ and PTEN loci. Interestingly, with regard to juvenile polyposis of infancy, the three index patients, aged between 7 months and 2 years at diagnosis, all carried BMPR1A PVs; only one, however, carried the 10q23 microdeletion while the other two harbored an exon 1 deletion and a splice site alteration (c.430+2T $>C)$, respectively. In SMAD4 PV carriers a more severe gastric phenotype as well as an HHT overlap phenotype were observed. In addition, index patients with SMAD4 missense PVs $(n=14)$ displayed a significantly younger median age at diagnosis (10.5 years, IQR 22.0) compared with those with SMAD4 frameshift changes (28.0 years, IQR $31.0, n=29 ; p=0.028$ ); this finding, however, was not present among nonindex patients (missense: 36 years, IQR 12.8; frameshift: 29 years, IQR 30.0; $p=0.39$ ). Even when combining the ECS and LBSB data sets no further statistically significant phenotypic differences with regard to the type of genetic variant or protein domain affected could be identified. We assume that this is partly due to the comparatively small numbers in the respective variant subgroups and partly to the pronounced phenotypic heterogeneity in JPS. The latter was particularly striking in carriers of identical germline variants (e.g., 19 patients from 9 unrelated families carrying the c.1244_1247del SMAD4 variant). Moreover, marked intrafamilial phenotypic heterogeneity, in particular regarding JP and cancer occurrence, was evident in four (1 SMAD4, 3 BMPR1A) 
Table 5 Surveillance recommendations in juvenile polyposis.

\begin{tabular}{|c|c|c|c|c|}
\hline Organ & Patients & Methods & Age at starting & Interval \\
\hline Colon, Rectum & & & & Depending on phenotype \\
\hline \multirow{2}{*}{ Stomach, Duodenum } & & & & Depending on phenotype \\
\hline & BMPR1A & & 25 years & 3 years \\
\hline Vascular manifestations & SMAD4 & Referral to HHT specialist & & \\
\hline
\end{tabular}

ECS families with data available on five to ten family members (data not shown).

\section{DISCUSSION}

This large comprehensive retrospective study on genotypephenotype correlations in JPS corroborates previous observations that SMAD4 carrier status is associated with HHT, multiple gastric JPs, and gastric cancer occurrence; provides recommendations for management on the basis of both new and previously published data (Table 5); and establishes the basis for open access SMAD4 and BMPR1A Leiden Open Variant Database-powered variant databases. ${ }^{19}$

The reported age at diagnosis of JPS was similar for both SMAD4 and BMPR1A pathogenic variant carriers (median 28 and 25 years, respectively). The youngest index patients reported, both in the ECS and the LBSB group (median age 1.5 and 6 years, respectively), consisted of carriers of large cytogenetic alterations at 10q23, e.g., microdeletions encompassing the BMPR1A and PTEN loci.

As expected, JPS-HHT symptoms in both the ECS and LBSB group were exclusively reported in SMAD4 but not in BMPR1A pathogenic variant carriers. In addition, anemia was twice as often observed in SMAD4 carriers with $32 \%$ displaying the hemorrhagic telangiectasia overlap syndrome (HHT) phenotype $(p=0.0006)$. Unfortunately, the true incidence of HHT/ JPS overlap in those with a SMAD4 PV cannot be established, as not all SMAD4 carriers underwent systematic screening for HHT. However, given that almost one third had features of HHT in this cohort, in addition to past publications suggesting very high (around 90\%) prevalence of JPS/HHT overlap in SMAD4 carriers, ${ }^{9}$ we recommend that all patients with a SMAD4 PV undergo routine screening for HHT, such as a oneoff thoracic computed tomography (CT) scan to screen for pulmonary arteriovenous malformations and contrast echocardiogram for aortopathy. Surveillance guidelines are not uniform across countries, and thus referring such patients to a local HHT specialist center would be advised (European centers can be identified at https://vascern.eu/expertise/rarediseases-wgs/hht-wg/).

While JPs of the colon were twice as frequently reported in ECS index patients compared with affected family members, rectal JPs were significantly less frequent than colonic ones $(32 \%$ vs. $72 \% ; p<0.01)$, independent of the gene affected.
The prevalence and number of gastric JPs, however, were significantly increased in both SMAD4 index and nonindex patients, and occurred roughly 20 years later compared with the colonic JPs (40 vs. 18 years; $p<0.0001)$. Upper GI endoscopy surveillance is recommended for patients with JPS. The data presented here are sufficient, when combined with published data, to support that upper GI surveillance should be tailored to the underlying genotype. It would be reasonable to recommend upper GI surveillance to start at age 18 years for SMAD4 carriers, with intervals ranging from 1 to 3 years depending on the number of polyps (Table 5). However, although approximately $18 \%$ of $B M P R 1 A$ carriers develop gastric polyps, they are usually smaller and gastric cancer has not been reported. In view of these findings, starting surveillance in this group by the age of 25 years and a surveillance interval of 3 years seems reasonable until further data are available.

Among the $11 \%$ to $15 \%$ of LBSB and ECS patients with cancer, colorectal cancer accounted for 58\% (SMAD4) and $88 \%$ (BMPR1A) of all cancers reported and occurred about 20 years later (median age 41 years) compared with the age at diagnosis of colonic JPs $(18.5$ years; $p<0.0001)$. Most patients' colorectal disease is likely to be manageable endoscopically. Recent pediatric guidelines produced on behalf of the European Society for Paediatric Gastroenterology Hepatology and Nutrition (ESPHGAN) outline an approach to colorectal surveillance, commencing at age 12-15 with surveillance interval being personalized according to the individual's phenotype. ${ }^{20}$ However, some patients will require surgical intervention for either endoscopically unmanageable disease, or the development of cancer. Where surgery is being contemplated, it should be personalized according to the individual's phenotype. Usually in the prophylactic setting, a (sub)total colectomy will be the procedure of choice, retaining the rectum, avoiding the need for pelvic dissection.

As with the prevalence of gastric JPs, gastric cancer (median age 44 years) was exclusively reported in SMAD4 PV carriers, accounting for $27 \%$ of SMAD4 tumors. These observations were essentially mirrored by the LBSB group. It is likely that gastric cancer occurrence in SMAD4 carriers is underestimated since seven ECS patients with massive JP burden had prophylactic gastric resection/gastrectomy at a median 
age of 49.5 years. Thus, more of these patients might have developed gastric cancer. It is difficult on the basis of current evidence to give firm recommendations regarding gastrectomy in SMAD4 carriers. There are no data regarding outcomes from endoscopic polypectomy in the stomach. Decisions will need to be made on a case-by-case basis. Those with advanced gastric polyposis are best seen in a center experienced in managing this condition. It is likely that the gastric polyps have a relatively indolent progression. However, in those with advanced polyposis in whom dysplasia is found, and in those whose stomachs cannot be adequately/ safely surveyed, a discussion through a specialist multidisciplinary team is required and then the option of prophylactic/risk-reducing surgery should be discussed with the patient. As always, in the setting of prophylactic, riskreducing surgery, retaining the best possible quality of life is essential and therefore this surgery should be reserved to highly specialized centers.

In this study small intestine polyps were reported in $15.7 \%$ $(20 / 127)$ of SMAD4 carriers and $3.2 \%$ (3/94) of BMPR1A carriers. It should be noted that we did not distinguish duodenal from ileal/jejunal polyps and it is likely that duodenal polyps predominate here. ${ }^{21}$ In addition, given that small bowel surveillance is not routinely recommended, the true incidence of small bowel polyps cannot be established. However, these data do support the recommendation that the small bowel should undergo evaluation for patients who develop symptoms or unexplained anemia, e.g., with videocapsule endoscopy (VCE), to look for underlying small bowel polyps and angioectasia.

The limitations of this study obviously include its retrospective nature and, inevitably when dealing with such a rare disorder, the possibility of ascertainment and/or selection bias. In addition, differences in patient data collection as well as completeness of medical records among the participating centers will impact on data quality and comparability. Furthermore, certain of the reported phenotypic features likely represent underestimations since a particular trait may go unnoticed if not specifically searched for, e.g., arteriovenous malformations in SMAD4-associated JPS-HHT, as reported by O'Malley et al. ${ }^{9}$

To circumvent these issues clearly, prospective, multicenterbased studies, as exemplified by the Prospective Lynch Syndrome Database (PLSD), are needed, ${ }^{22}$ which could also inform the outcomes of surveillance measures taken. ${ }^{23}$ Furthermore, with the novel genetic high-throughput sequencing tools available, the roughly $50 \%$ of JPS patients and families in whom no SMAD4 or BMPR1A alteration can be identified constitute an obvious target for candidate gene analysis.

\section{SUPPLEMENTARY INFORMATION}

The online version of this article (https://doi.org/10.1038/s41436020-0826-1) contains supplementary material, which is available to authorized users.

\section{ACKNOWLEDGEMENTS}

We thank the patients and their families for participation in the study and Sophie Grandjouan for contributing clinical data. D.G.E. is supported through the National Institute for Health Research (NIHR) Manchester Biomedical Research Center (IS-BRC1215-20007). K.H. is supported by grants from the Stiftung zur Krebsbekaempfung (number 285) and the Freiwillige Akademische Gesellschaft.

\section{DISCLOSURE}

The authors declare no conflicts of interest.

Publisher's note Springer Nature remains neutral with regard to jurisdictional claims in published maps and institutional affiliations.

\section{REFERENCES}

1. Burt RW, Bishop DT, Lynch HT, Rozen P, Winawer SJ. Risk and surveillance of individuals with heritable factors for colorectal cancer. Bull World Health Organ. 1990;68:655.

2. Chow E, Macrae F. Review of juvenile polyposis syndrome. J Gastroenterol Hepatol. 2005;20:1634-1640.

3. Boland $C R$, Yurgelun MB. Historical perspective on familial gastric cancer. Cell Mol Gastroenterol Hepatol. 2017;3:192-200.

4. Larsen Haidle J, Howe JR. Juvenile polyposis syndrome. In: Adam MP, Ardinger $\mathrm{HH}$, Pagon RA, et al., editors. GeneReviews. Seattle, WA: University of Washington; 1993.

5. Howe JR, Sayed MG, Ahmed AF, et al. The prevalence of MADH4 and BMPR1A mutations in juvenile polyposis and absence of BMPR2, BMPR1B, and ACVR1 mutations. J Med Genet. 2004;41:484-491.

6. Aretz $S$, Stienen D, Uhlhaas $S$, et al. High proportion of large genomic deletions and a genotype-phenotype update in 80 unrelated families with juvenile polyposis syndrome. J Med Genet. 2007;44:702-709.

7. Calva-Cerqueira D, Chinnathambi S, Pechman B, Bair J, Larsen-Haidle J, Howe JR. The rate of germline mutations and large deletions of SMAD4 and BMPR1A in juvenile polyposis. Clin Genet. 2009;75:79-85.

8. Zhao M, Mishra L, Deng C-X. The role of TGF-beta/SMAD4 signaling in cancer. Int J Biol Sci. 2018;14:111-123.

9. O'Malley M, LaGuardia L, Kalady MF, et al. The prevalence of hereditary hemorrhagic telangiectasia in juvenile polyposis syndrome. Dis Colon Rectum. 2012;55:886-892.

10. Starr LJ, Lindor NM, Lin AE. Myhre syndrome. In: Adam MP, Ardinger HH, Pagon RA, et al., editors. GeneReviews. Seattle, WA: University of Washington; 1993.

11. Dahdaleh FS, Carr JC, Calva D, Howe JR. Juvenile polyposis and other intestinal polyposis syndromes with microdeletions of chromosome 10q22-23. Clin Genet. 2012;81:110-116.

12. Howe JR, Roth S, Ringold JC, et al. Mutations in the SMAD4/DPC4 gene in juvenile polyposis. Science. 1998;280:15-18.

13. Woodford-Richens K, Williamson J, Bevan S, et al. Allelic loss at SMAD4 in polyps from juvenile polyposis patients and use of fluorescence in situ hybridization to demonstrate clonal origin of the epithelium. Cancer Res. 2000;60:2477-2482.

14. Langeveld D, van Hattem WA, de Leng WWJ, et al. SMAD4 immunohistochemistry reflects genetic status in juvenile polyposis syndrome. Clin Cancer Res. 2010;16:4126-4134.

15. Blatter RHE, Plasilova M, Wenzel F, et al. Somatic alterations in juvenile polyps from BMPR1A and SMAD4 mutation carriers. Genes Chromosomes Cancer. 2015;54:575-582.

16. McColl I, Busxey HJ, Veale AM, Morson BC. Juvenile polyposis coli. Proc R Soc Med. 1964;57:896-897.

17. Syngal S, Brand RE, Church JM, Giardiello FM, Hampel HL, Burt RW $A C G$ clinical guideline: genetic testing and management of hereditary gastrointestinal cancer syndromes. Am J Gastroenterol. 2015;110: 223-262.

18. Richards S, Aziz N, Bale S, et al. Standards and guidelines for the interpretation of sequence variants: a joint consensus recommendation of the American College of Medical Genetics and Genomics and the Association for Molecular Pathology. Genet Med. 2015;17:405-423. 
19. Fokkema IFAC, Taschner PEM, Schaafsma GCP, Celli J, Laros JFJ, den Dunnen JT. LOVD v.2.0: the next generation in gene variant databases. Hum Mutat. 2011:32:557-563.

20. Cohen S, Hyer W, Mas E, et al. Management of juvenile polyposis syndrome in children and adolescents: a position paper from the ESPGHAN Polyposis Working Group. J Pediatr Gastroenterol Nutr. 2019; 68:453-462.

21. Postgate AJ, Will OC, Fraser CH, Fitzpatrick A, Phillips RKS, Clark SK. Capsule endoscopy for the small bowel in juvenile polyposis syndrome: a case series. Endoscopy. 2009;41:1001-1004.

22. Møller P, Seppälä TT, Bernstein I, et al. Cancer risk and survival in path_MMR carriers by gene and gender up to 75 years of age: a report from the Prospective Lynch Syndrome Database. Gut. 2018;67:1306-1316.

23. Latchford AR, Neale K, Phillips RKS, Clark SK. Juvenile polyposis syndrome: a study of genotype, phenotype, and long-term outcome. Dis Colon Rectum. 2012;55:1038-1043.
Open Access This article is licensed under a Creative Commons (c) (i) (2) $\Theta$ Attribution-NonCommercial-NoDerivatives 4.0 International License, which permits any non-commercial use, sharing, distribution and reproduction in any medium or format, as long as you give appropriate credit to the original author(s) and the source, and provide a link to the Creative Commons license. You do not have permission under this license to share adapted material derived from this article or parts of it. The images or other third party material in this article are included in the article's Creative Commons license, unless indicated otherwise in a credit line to the material. If material is not included in the article's Creative Commons license and your intended use is not permitted by statutory regulation or exceeds the permitted use, you will need to obtain permission directly from the copyright holder. To view a copy of this license, visit http://creativecommons.org/licenses/by-nc-nd/4.0/.

(C) The Author(s) 2020 